\title{
High efficiency planar geometry germanium-on-silicon single-photon avalanche diode detectors
}

Thorburn, Fiona, Huddleston, Laura, Kirdoda, Jarosław, Millar, Ross, Ferre-Llin, Lourdes, et al.

Fiona E. Thorburn, Laura L. Huddleston, Jarosław Kirdoda, Ross W. Millar, Lourdes Ferre-Llin, Xin Yi, Douglas J. Paul, Gerald S. Buller, "High efficiency planar geometry germanium-on-silicon single-photon avalanche diode detectors," Proc. SPIE 11386, Advanced Photon Counting Techniques XIV, $113860 \mathrm{~N}$ (18 May 2020); doi: 10.1117/12.2559620

Event: SPIE Defense + Commercial Sensing, 2020, Online Only, California, United States 


\title{
High Efficiency, Planar Geometry Germanium-on-Silicon Single- Photon Avalanche Diode Detectors
}

\author{
Fiona E. Thorburn ${ }^{\mathrm{a}}$, Laura L. Huddleston ${ }^{\mathrm{a}}$, Jarosław Kirdoda ${ }^{\mathrm{b}}$, Ross W. Millar ${ }^{\mathrm{b}}$, \\ Lourdes Ferre-Llin ${ }^{\mathrm{b}}$, Xin Yi ${ }^{\mathrm{a}}$, Douglas J. Paul ${ }^{\mathrm{b}}$, Gerald S. Buller*a \\ anstitute of Photonics and Quantum Sciences, School of Engineering and Physical Sciences, Heriot- \\ Watt University, Edinburgh, EH14 4AS, United Kingdom; \\ bJames Watt School of Engineering, University of Glasgow, Rankine Building, Oakfield Avenue, \\ Glasgow, G12 8LT, United Kingdom
}

\begin{abstract}
This paper presents the performance of $26 \mu \mathrm{m}$ and $50 \mu$ m diameter planar Ge-on-Si single-photon avalanche diode (SPAD) detectors. The addition of germanium in these detectors extends the spectral range into the short-wave infrared (SWIR) region, beyond the capability of already well-established Si SPAD devices. There are several advantages for extending the spectral range into the SWIR region including: reduced eye-safety laser threshold, greater attainable ranges, and increased depth resolution in range finding applications, in addition to the enhanced capability to image through obscurants such as fog and smoke. The time correlated single-photon counting (TCSPC) technique has been utilized to observe record low dark count rates, below $100 \mathrm{kHz}$ at a temperature of $125 \mathrm{~K}$ for up to a $6.6 \%$ excess bias, for the $26 \mu \mathrm{m}$ diameter devices. Under identical experimental conditions, in terms of excess bias and temperature, the $50 \mu \mathrm{m}$ diameter device consistently demonstrates dark count rates a factor of 4 times greater than $26 \mu \mathrm{m}$ diameter devices, indicating that the dark count rate is proportional to the device volume. Single-photon detection efficiencies of up to $\sim 29 \%$ were measured at a wavelength of $1310 \mathrm{~nm}$ at $125 \mathrm{~K}$. Noise equivalent powers (NEP) down to $9.8 \times 10^{-17} \mathrm{WHz}^{-1 / 2}$ and jitters < $160 \mathrm{ps}$ are obtainable, both significantly lower than previous $100 \mu \mathrm{m}$ diameter planar geometry devices, demonstrating the potential of these devices for highly sensitive and high-speed imaging in the SWIR.
\end{abstract}

Keywords: Single-photon, Lidar, Avalanche photodiodes, Photodetectors

\section{INTRODUCTION}

Devices which are capable of the detection of single-photons using impact ionization in avalanche diodes have become vital for in several emerging applications areas. These applications include Light Detection and Ranging (LiDAR) and 3D imaging using time-of-flight ${ }^{1-3}$ or range-gating ${ }^{4}$, quantum key distribution (QKD) ${ }^{5}$, fiber/free space quantum communications ${ }^{6,7}$ and quantum optics ${ }^{8}$. CMOS based single-photon avalanche diode (SPAD) detectors have been commercially available for a number of years and are well-established as highly-sensitive, large area detector arrays with integrated electronics ${ }^{9,10}$. The drawback of these devices, however, is that they are limited to operation below wavelengths of $\lambda \sim 1 \mu \mathrm{m}$ because of the wide bandgap of Si. There are many advantages for extending the operating range of such devices beyond this limit and into the short-wave infrared (SWIR) spectral region. The ability to operate at the low-loss optical fiber telecommunications wavelengths of $1310 \mathrm{~nm}$ and $1550 \mathrm{~nm}$ is clearly essential for quantum communications applications which require high-speed and ultra-sensitive SWIR SPAD detectors. Free space applications, such as LiDAR, can also benefit greatly from operating in the SWIR because the eye-safety laser threshold increases substantially in this spectral range compared to wavelengths $<1 \mu \mathrm{m}$, thus much higher power optical sources can be utilized in active imaging resulting in improved depth resolution and longer obtainable maxi mum range ${ }^{11}$. In addition to this, solar radiation which considerably increases the background level in many SPAD based LIDAR applications is significantly reduced in the SWIR ${ }^{12}$. Finally, atmospheric transmission is greatly improved particularly when imaging through obscurants including, for example: smoke, dust, haze and snow, due to decreased Mie scattering in the SWIR spectral region ${ }^{13,14}$. In the SWIR spectral region, the two most commonly used single-photon detectors are InGaAs/InP based SPADs and superconducting nanowire detectors. The latter provide particularly high performance for single-photon detection [15],

*g.s.buller@hw.ac.uk; single-photon.com

Advanced Photon Counting Techniques XIV, edited by Mark A. Itzler, Joshua C. Bienfang,

K. Alex Mclntosh, Proc. of SPIE Vol. 11386, 113860N · c) 2020 SPIE

CCC code: $0277-786 \mathrm{X} / 20 / \$ 21 \cdot$ doi: $10.1117 / 12.2559620$ 
however, their practicality is limited for some applications due to cryogenic cooling requirements, as they are typically operated at temperatures less than $3 \mathrm{~K}$. InGaAs/InP SPADs have been extensively developed to the point that highperformance imaging array cameras have been developed and demonstrated in several single-photon depth imaging applications $3,15-17$. Typically, InGaAs/InP based SPADs exhibit single-photon detection efficiencies (SPDEs) ranging from $20 \%$ to $>50 \%$ in the SWIR and operate at Peltier-cooled temperatures between $220 \mathrm{~K}$ and $255 \mathrm{~K}^{18-23}$, this is advantageous because Peltier-cooling has less stringent requirements for size, weight and power consumption in comparison to cryogenic cooling, facilitating the development of compact detector modules. Another challenge associated with InGaAs based SPADs is that the achievable count rates are markedly restricted by prominent afterpulsing effects ${ }^{24-26}$, however, progress continues to be made in mitigating this issue ${ }^{21,27}$.

An alternative to these methods is to use germanium as an absorber; the bandgap of Ge allows for efficient photon absorption at wavelengths up to $\sim 1600 \mathrm{~nm}$ at room temperature. Dual temperature growth techniques have resulted in high quality Ge layers grown on $\mathrm{Si}^{28}$, facilitating the fabrication of optical detectors capable of absorption potentially up to $\lambda \sim 1600 \mathrm{~nm}$ and the potential to be fabricated in Si foundries. Effective and reliable fabrication techniques have made possible many demonstrations of Ge-on-Si PIN diodes and linear avalanche photodetectors with impressive

performance ${ }^{28-30}$. The first single-photon detector using a Ge-containing absorber adjacent to a Si multiplication layer was reported in 2002 by Loudon et al. in which they utilized a $\mathrm{SiGe} / \mathrm{Si}$ multiple quantum well structure to absorb $1210 \mathrm{~nm}$ wavelength photons ${ }^{31}$. In $2011 \mathrm{Lu}$ et al. presented a Ge-on-Si SPAD with SPDE up to $14 \%$ and dark count rates (DCRs) of the order of 100s of Mcps (counts per second) at a temperature of $200 \mathrm{~K}$, for a wavelength of $1310 \mathrm{~nm}{ }^{32}$. Shortly after, Warburton et al. reported a mesa etched design which at the same wavelength obtained an SPDE of $4 \%$ and DCR of $6 \mathrm{Mcps}$ at $100 \mathrm{~K}^{24}$, and also for the first time demonstrated single-photon detection at $1550 \mathrm{~nm}$ from a Si based device. The disadvantage of the mesa geometry associated with the SPADs reported in ${ }^{24,32}$, is that the exposed sidewalls of the devices contribute significantly to the DCR, resulting in high values of the order of Mcps, which places serious restrictions on the device performance. Martinez et al. demonstrated a solution to this in 2017 through the use of a waveguide coupled Ge-on-Si SPAD at $80 \mathrm{~K}$, which exhibited an SPDE of $5.27 \%$ and much improved DCR of $534 \mathrm{kcps}$ at a wavelength of $1310 \mathrm{~nm}^{33}$. Another alternative design which avoids the limitations imposed by the exposed sidewalls is that of a planar geometry design ensuring that the high field region is maintained far from any sidewall. We previously reported a planar device with an active area diameter of $100 \mu \mathrm{m}$ exhibiting an impressive SPDE of $38 \%$ at $1310 \mathrm{~nm}$ for a temperature of $125 \mathrm{~K}$. Due to a relatively large diameter, however, the device exhibited a rather high DCR of $2 \mathrm{Mcps}{ }^{25}$. A planar geometry $100 \mu \mathrm{m}$ diameter Ge-on-Si SPAD was subsequently utilized for laboratory-based-LiDAR experiments at short ranges which allowed modelling of performance under more realistic scenarios of operation ${ }^{34}$, clearly demons trating the potential of these devices in 3D imaging applications.

In this paper, we report on the performance of $26 \mu \mathrm{m}$ and $50 \mu \mathrm{m}$ diameter planar Ge-on-Si SPADs and compare the results to prior planar devices. A significant reduction in the DCR is observed with these smaller area devices and we conclude that the DCR scales proportionally with volume. SPDEs up to $\sim 29 \%$ are demonstrated at $125 \mathrm{~K}$ for a wavelength of $1310 \mathrm{~nm}$, although less than the $38 \%$ reported for a larger $100 \mu \mathrm{m}$ diameter device ${ }^{25}$, it is proposed that this is limited by experimental design rather than the device itself. The particularly low DCR displayed by the $26 \mu \mathrm{m}$ diameter detector indicates a highly sensitive single-photon detector, quantified by a noise equivalent power (NEP) of $9.8 \times 10^{-17} \mathrm{WHz}^{-1 / 2}$; seven times lower than that observed from the $100 \mu \mathrm{m}$ diameter planar geometry devices, at the same temperature. Furthermore, low jitter performance is demonstrated; down to $157 \pm 10 \mathrm{ps}$.

\section{EXPERIMENTAL PROCEDURE}

\subsection{Device fabrication}

The SPADs were grown on $150 \mathrm{~mm}$ diameter $\mathrm{n}^{++}-\mathrm{Si}(001)$ substrates. First, a $1.5 \mu \mathrm{m}$ thickness of nominally undoped $\mathrm{Si}$ was grown epitaxially using a commercial reduced pressure chemical vapor deposition (RPCVD) system. Subsequently, photolithography was used to define windows for the charge sheet layers for each SPAD device. The doping level and thickness of the charge sheet layer controls the relative electric field profiles in the Ge absorber and Si avalanche region. The devices were implanted using boron at $10 \mathrm{keV}$ and activated with a $30 \mathrm{~s}$ anneal at $950^{\circ} \mathrm{C}$. The wafers were cleaned, 
then $1 \mu \mathrm{m}$ of undoped Ge was grown before a $50 \mathrm{~nm} \mathrm{p}^{++}$-Ge cap which constitutes the top contact. The $\mathrm{p}^{++}-\mathrm{Ge}$ top contact area was etched using photolithography and a fluorine inductively coupled plasma reactive ion etch (ICP-RIE). Photolithography was also used to define trenches between the SPAD devices and a fluorine ICP-RIE etched through the Ge epilayers to provide lateral electrical isolation. The device was planarized with hydrogen silsesquioxane (HSQ) and plasma enhanced chemical vapor deposition (PECVD) $\mathrm{SiO}_{2}$. Next, Ti/Al was evaporated onto the backside of the wafers and annealed to form a bottom Ohmic contact to the $\mathrm{n}^{++}-\mathrm{Si}$ substrate followed by photolithography again to form holes to the $\mathrm{p}^{++}$cap, then Ti/Al was deposited to form an Ohmic contact to the top of the device. Lastly, a silicon nitride antireflection coating was deposited by PECVD on top of the device to reduce surface reflections. A detailed description of the whole process can be found in ${ }^{25}$. A cross-section of a single planar geometry Ge-on-Si SPAD is shown in Figure 1(a) and a microscope image of the top view of a chip containing planar Ge-on-Si SPADs is shown in Figure 1(b).

(a)

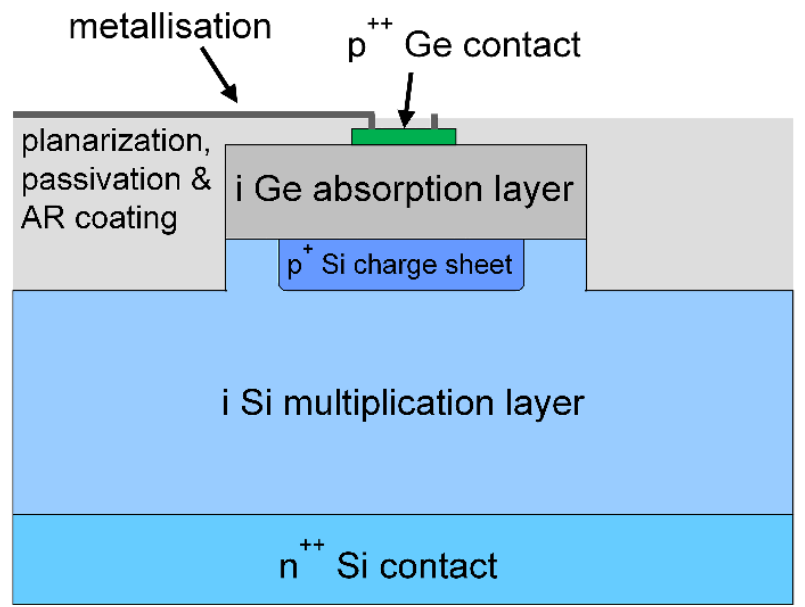

(b)

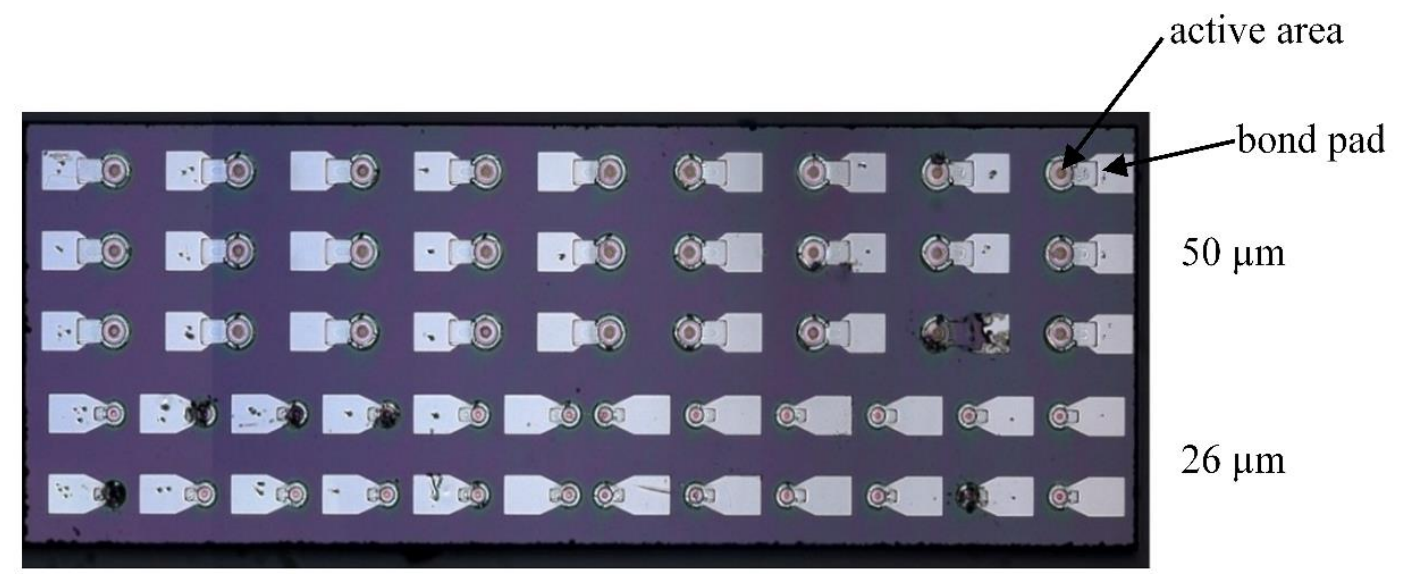

Figure 1. (a) A cross-section of a planar Ge-on-Si SPAD. This diagram shows the silicon bottom contact, multiplication and charge sheet layers, as well as the germanium absorption and top contact layers and finally, the planarization, passivation, AR coating and metallization layers $(\mathrm{i}=$ intrinsic). (b)A microscope image at $\times 5$ magnification of a chip containing three rows of $50 \mu \mathrm{m}$ diameter and 2 rows of $26 \mu \mathrm{m}$ diameter Ge-on-Si SPADs, the metal bond pad and device active area are highlighted.

\subsection{Detector characterization using time-correlated single-photon counting (TCSPC)}

A schematic diagram of the experimental set-up used to measure the SPDE, DCR and jitter parameters is shown in Figure 2 . 


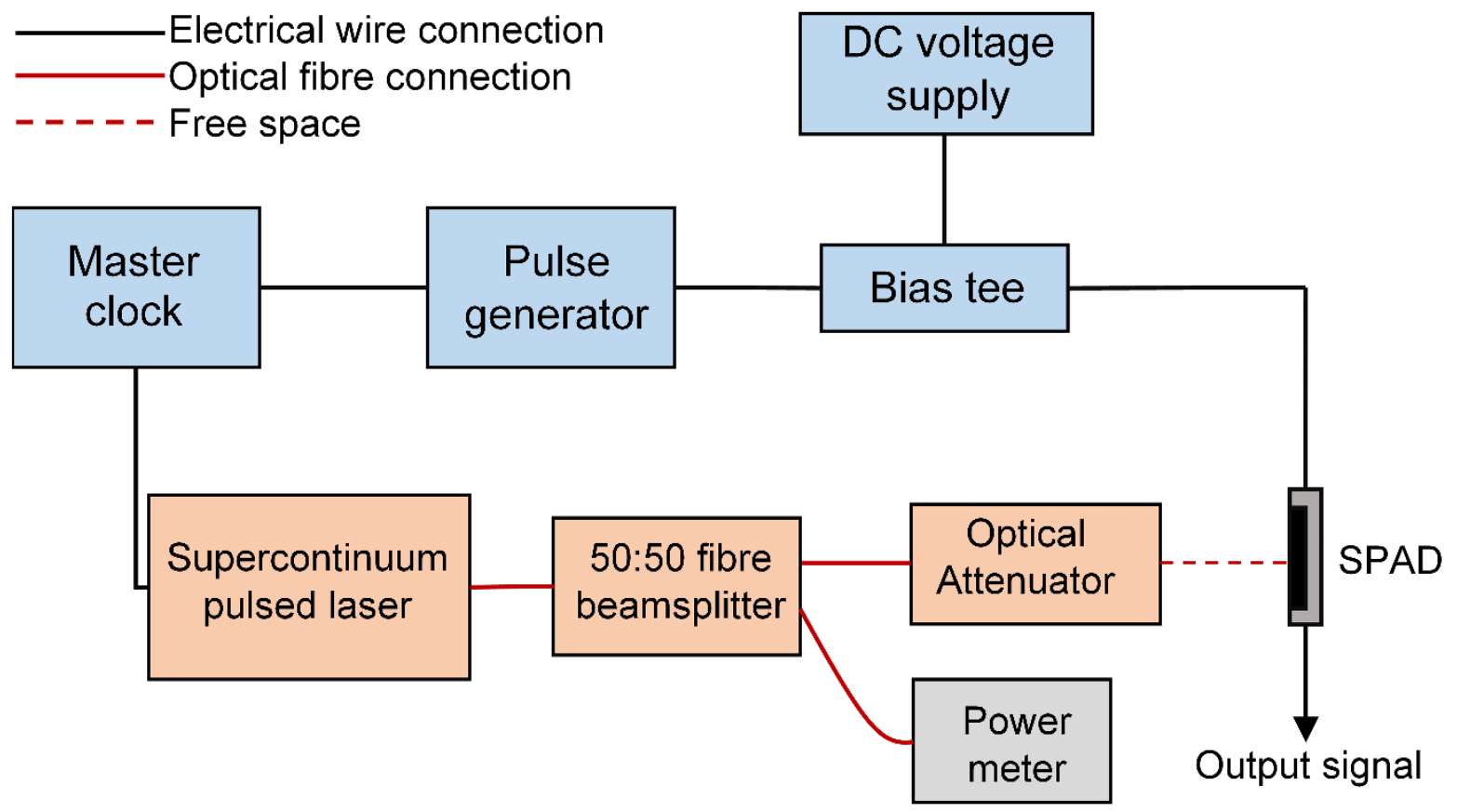

Figure 2. A schematic diagram of the TCSPC experimental set-up. The SPAD was mounted in a variable temperature cryostat on a translation stage with 3D movement. The optical alignment of the focused attenuated beam onto the detector active area was performed using an illuminated SWIR camera arrangement (not shown in the diagram).

The chip containing the SPADs was mounted in a variable temperature Oxford Instruments cryostat which was maintained at a temperature of $125 \mathrm{~K}$ for these measurements. The laser used was a pulsed NKT Supercontinuum laser, tunable in the wavelength range of $1150-2000 \mathrm{~nm}$. The laser's output was coupled into a single mode optical fiber and directed into an in-fiber 50:50 beam splitter. One output from the splitter was measured by a calibrated power meter to allow continuous monitoring of the power level to the optical system. The other output was directed to through a calibrated optical attenuator with a maximum attenuation of $100 \mathrm{~dB}$, and then a lens focused the output from this onto the device active area. A second, removable, power meter was utilized to measure the power after the lens and thus calculate the attenuation required to result in less than 0.01 photons per pulse incident upon the SPAD, ensuring the probability of $>1$ photon per pulse was statistically negligible. A fixed direct current (DC) voltage at a level just below the avalanche breakdown voltage was applied to the device under investigation and a gated voltage supplied by the pulse generator was applied to periodically take the device above the avalanche breakdown level for a gate of $\sim 50 \mathrm{~ns}$. This gated signal was applied in synchronization with the incident optical attenuated pulses from the laser, allowing the SPAD to operate in the Geiger mode whilst the photons are incident. Outside of the gating pulses, the detector was held below avalanche breakdown until the next optical pulse was incident. The cathode of the SPAD was connected to the photon counting data acquisition unit and the measurements were taken at a low repetition rate of $10 \mathrm{kHz}$ to minimize the probability of afterpulsing affecting the measurements.

\section{RESULTS}

The SPDE and DCR for both the $26 \mu \mathrm{m}$ and $50 \mu \mathrm{m}$ diameter devices are displayed in Figures 3(a) and (b) respectively as a function of excess bias. 

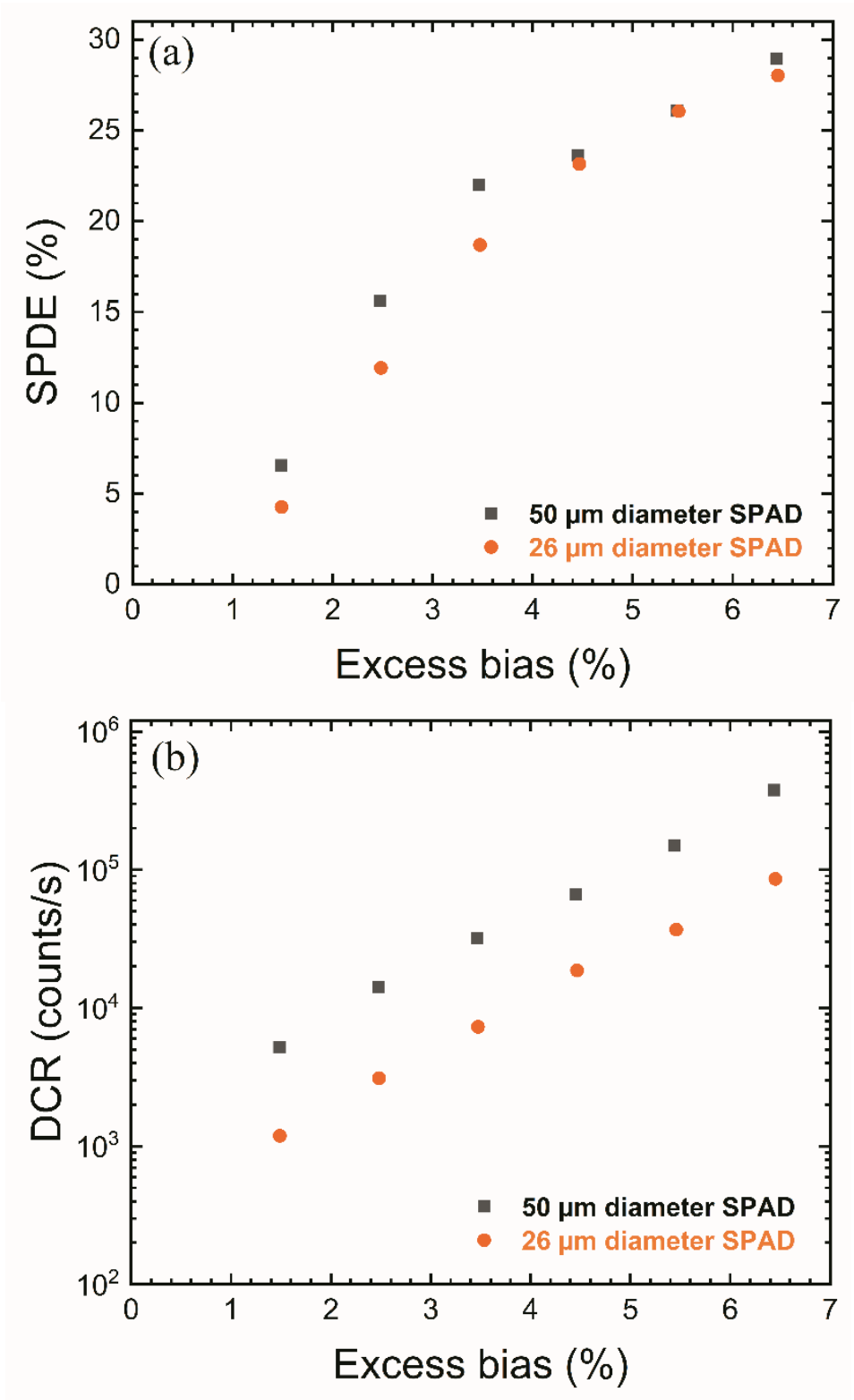

Figure 3. (a) The SPDE and (b) DCR of the $26 \mu \mathrm{m}$ and $50 \mu \mathrm{m}$ diameter Ge-on-Si SPADs as a function of excess bias at a temperature of $125 \mathrm{~K}$. The SPDE values were measured at an incident photon wavelength of $1310 \mathrm{~nm}$.

As can be observed in Figure 3(a), the maximum SPDEs measured were $28 \%$ and $29 \%$ for the $26 \mu \mathrm{m}$ and $50 \mu \mathrm{m}$ diameter devices respectively, at a $6.5 \%$ excess bias. This is $10 \%$ lower than that of the $100 \mu \mathrm{m}$ device reported previously, it is proposed that this may be partly because the $26 \mu \mathrm{m}$ and $50 \mu \mathrm{m}$ diameter devices at present may not collect all photons during the SPDE measurements. Further work is needed in order to identify any other mechanisms which may account for the decrease in SPDE for these smaller area devices. In Figure 3(b), it can be seen that the DCR for the $26 \mu \mathrm{m}$ device remains below $100 \mathrm{kcps}$ for excess bias up to the maximum of $6.5 \%$. The DCR for the larger $50 \mu \mathrm{m}$ device is consistently $\sim 4$ times greater than for the smaller device, reaching a maximum of $\sim 380 \mathrm{kcps}$ for an excess bias of $6.5 \%$. This is 4.4 times greater than the DCR for the $26 \mu \mathrm{m}$ device at the same bias, which measured $\sim 86 \mathrm{kcps}$. These results suggest that the DCR is proportional to the device volume, indicating that surface contributions to the total DCR are minimal. With 
reference to our previous work ${ }^{25}$, under the same experimental conditions in terms of wavelength and temperature, the DCRs reported for the $100 \mu \mathrm{m}$ diameter planar device vary between 13.5 and 51 times greater than the $50 \mu \mathrm{m}$ DCR levels reported here, up to an excess bias of $5.5 \%$. Although it may be expected that the DCR for the $100 \mu \mathrm{m}$ diameter device should again be 4 times greater for twice the diameter, the former device was fabricated much earlier and the fabrication processes where significantly improved since, thus this has led to much improved DCR levels.

The NEP is a useful parameter for indicating the sensitivity of a SPAD detector and it is calculated from the SPDE and DCR using equation (1)

$$
N E P=\frac{h v}{S P D E} \sqrt{2 D C R}
$$

where $h$ represents Planck's constant and $v$ is the frequency of the incident photons. The NEP is useful for comparing detectors, with lower values indicating higher optical sensitivity. For the $26 \mu \mathrm{m}$ diameter device, the minimum NEP was calculated as $9.8 \times 10^{-17} \mathrm{WHz}^{-1 / 2}$ at an excess bias of $\sim 3.5 \%$ and for the $50 \mu \mathrm{m}$ device the lowest NEP calculated was $1.6 \times 10^{-16} \mathrm{WHz}^{-1 / 2}$ recorded at an excess bias of $\sim 2.5 \%$. These figures represent a 7 and 4 fold reduction in this parameter respectively compared the $100 \mu \mathrm{m}$ diameter SPAD in ${ }^{25}$. Whilst not as low as that of InGaAs/InP SPADs which have demonstrated NEP values down to $1 \times 10^{-17} \mathrm{WHz}^{1 / 2}$ for $\lambda=1550 \mathrm{~nm}$ at $223 \mathrm{~K}$, these devices have shown considerable improvement compared to mesa geometry and larger area planar Ge-on-Si SPADs. Thus, work is ongoing to improve these devices by reducing the DCR to allow operation at higher temperatures consistent with Peltier-cooling.

The jitter of each device was measured as a function of excess bias and is expressed as the full-width-at-half-maximum of the timing histogram recorded in each case. The minimum jitter measured was $157 \pm 10 \mathrm{ps}$, this was recorded for the $26 \mu \mathrm{m}$ diameter device at an excess bias of $6.5 \%$ and the timing histogram is displayed in Figure 4 (a). Figure 4 (b) shows the jitter measured for both the $26 \mu \mathrm{m}$ and $50 \mu \mathrm{m}$ diameter devices as a function of the excess bias, as expected the jitter decreases with increasing excess bias. The $50 \mu \mathrm{m}$ diameter device exhibited a minimum jitter of $\sim 210 \pm 10 \mathrm{ps}$ for an excess bias of $6.5 \%$. The jitter reported for the $100 \mu \mathrm{m}$ diameter planar SPAD in ${ }^{25}$ was $310 \mathrm{ps}$ for an excess bias of $5.5 \%$, measured at a lower temperature of $78 \mathrm{~K}$. Therefore, the smaller area detectors improve the speed capability of the device as seen in other material systems, as a result of the restricted lateral extent of the avalanche build-up ${ }^{35}$.

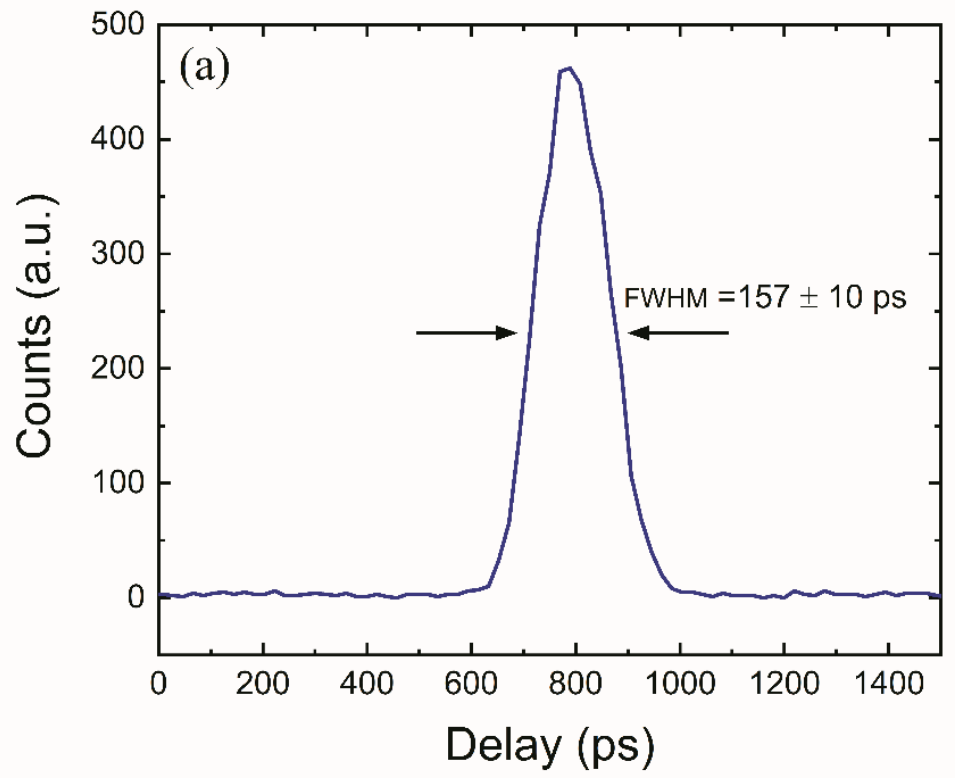

Proc. of SPIE Vol. 11386 113860N-6 


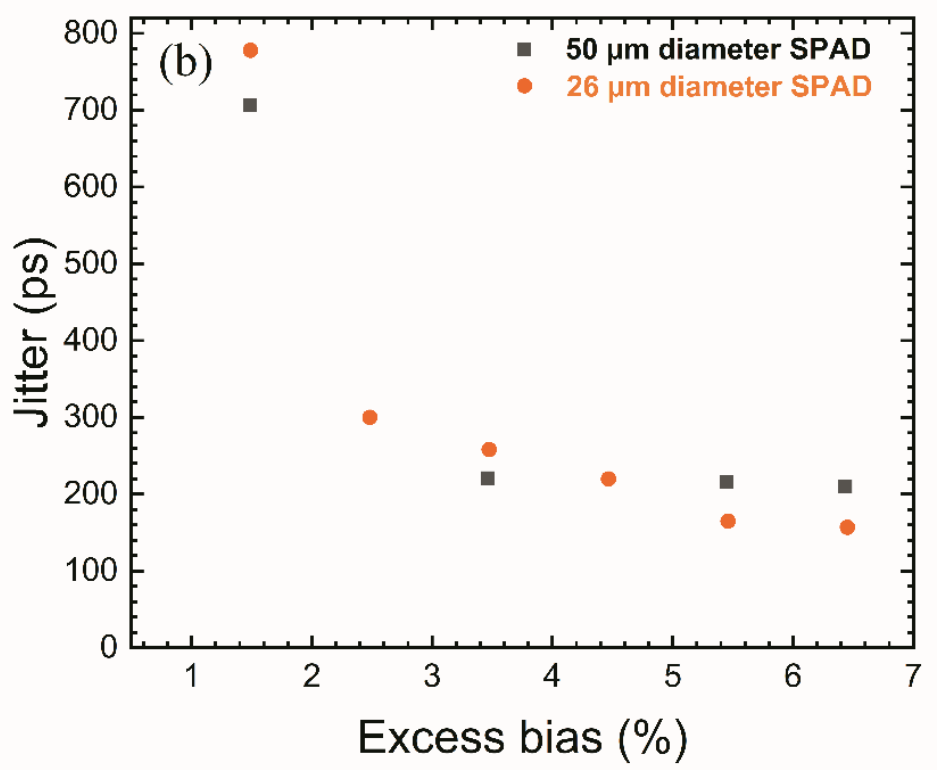

Figure 4. (a) Timing histogram for the minimum jitter recorded, this was obtained by the $26 \mu \mathrm{m}$ diameter device at an excess bias of $6.5 \%$. (b) Temporal jitter, expressed as full-width-at-half-maximum, of the $26 \mu \mathrm{m}$ and $50 \mu \mathrm{m}$ diameter Ge-on-Si SPADs as a function of excess bias at a temperature $125 \mathrm{~K}$ for an incident wavelength of $1310 \mathrm{~nm}$.

\section{CONCLUSIONS}

In summary, the performance of $26 \mu \mathrm{m}$ and $50 \mu \mathrm{m}$ diameter planar geometry Ge-on-Si SPADs has been investigated. At a wavelength of $1310 \mathrm{~nm}$ and temperature of $125 \mathrm{~K}$, the $26 \mu \mathrm{m}$ and $50 \mu \mathrm{m}$ diameter SPADs exhibited maximum SPDE values of $28 \%$ and $29 \%$ respectively. The DCR observed from the $26 \mu \mathrm{m}$ device was extremely low; staying below $100 \mathrm{kcps}$ for an excess bias up to $6.5 \%$. The $50 \mu \mathrm{m}$ diameter device DCR was approximately 4 times greater than $26 \mu \mathrm{m}$ device at each excess bias level recorded and so proportional to the device volume. This preliminary result indicates that surface contributions to the overall DCR are minimal, an important result to help our understanding of the devices and developing the design and fabrication processes going forward. High-speed operation was also observed; the smaller diameter device exhibited the best performance with jitter down to $157 \pm 10 \mathrm{ps}$, the results conclusively show that smaller devices reduce the device jitter as previously seen in SPADs fabricated in other material systems. Furthermore, highsensitivity operation was also observed, this is quantified by a minimum NEP value of $9.8 \times 10^{-17} \mathrm{WHz}^{-1 / 2}$ for the $26 \mu \mathrm{m}$ diameter device representing a 7 fold reduction compared to a larger $100 \mu$ m diameter planar Ge-on-Si SPAD under similar experimental conditions. The results presented show that utilizing smaller diameter devices significantly improves the performance of Ge-on-Si Geiger mode detectors demonstrating the potential for highly efficient, fast and affordable singlephoton detectors in the SWIR for a variety of applications.

\section{ACKNOWLEDGEMENTS}

The authors acknowledge the support of UK Engineering and Physical Sciences Research Council (EPSRC) projects UK EPSRC (EP/N003446/1, EP/L024020/1, EP/N003225/1, EP/S026428/1, EP/T00097X/1, EP/T001011/1); InnovateUK (104615); UK Royal Academy of Engineering Research Fellowship Scheme (Project RF-201819-18-187).

\section{REFERNCES}

[1] Pawlikowska, A. M., Halimi, A., Lamb, R. A. \& Buller, G. S., "Single-photon three-dimensional imaging at up to 10 kilometers range," Opt. Express 25(10), 11919-11931 (2017). 
[2] Tobin, R., Halimi, A., McCarthy, A., Ren, X., McEwan, K., McLaughlin, S. and Buller, G. S., "Long-range depth profiling of camouflaged targets using single-photon detection," Optical Engineering 57(3), 031303 (2017).

[3] Tachella, J., Altmann, Y., Mellado, N., McCarthy, A., Tobin, R., Buller, G. S., Tourneret, J. and McLaughlin, S., "Real-time 3D reconstruction from single-photon lidar data using plug-and-play point cloud denoisers," Nat. Commun. 10, 4984 (2019).

[4] Ren, X., Connolly, P. W. R., Halimi, A., Altmann, Y., McLaughlin, S., Gyongy, I., Henderson, R. K. and Buller, G. S., "High-resolution depth profiling using a range-gated CMOS SPAD quanta image sensor," Opt. Express 26(5), 5541-5557 (2018).

[5] Takesue, H., Nam, S. W., Zhang, Q., Hadfield, R. H., Honjo, T., Tamaki, K. and Yamamoto, Y., "Quantum key distribution over a 40-dB channel loss using superconducting single-photon detectors," Nat. Photonics 1, 343348 (2007).

[6] Gordon, K. J., Fernandez, V., Townsend, P. D. and Buller, G. S., "A short wavelength GigaHertz clocked fiberoptic quantum key distribution system," IEEE J. Quantum Electron. 40(7), 900-908 (2004).

[7] Pugh, C. J., Kaiser, S., Bourgoin, J., Jin, J., Sultana, N., Agne, S., Anisimova, E., Makarov, V., Choi, E. and Higgins, B. L., "Airborne demonstration of a quantum key distribution receiver payload," Quantum Sci. Technol. 2(2), 024009 (2017).

[8] Tanzilli, S., Martin, A., Kaiser, F., De Micheli, M. P., Alibart, O. and Ostrowsky, D. B., "On the genesis and evolution of Integrated Quantum Optics," Laser Photonics Rev. 6(1), 115-143 (2012).

[9] Rech, I., Gulinatti, A., Zappa, F., Ghiono, M and Cova, S., "High-performance silicon single-photon avalanche diode array," Proc. SPIE 7320, 73200H (2009).

[10] Gyongy, I., Calder., N, Davies, A., Dutton, N. A. W., Duncan, R. R., Rickman, C., Dalgarno, P. and Henderson, R. K., "A 256 x 256 , 100-kfps, 61\% Fill-Factor SPAD Image Sensor for Time-Resolved Microscopy Applications," IEEE Trans. Electron Devices 65(2), 547-554 (2018).

[11] Wallace, A. M., Halimi, A. and Buller, G. S., "Full waveform LiDAR for adverse weather conditions," IEEE Trans. Veh. Technol., doi:10.1109/TVT.2020.2989148 (2020).

[12] Bird, R. E., Hulstrom, R. L. and Lewis, L. J., "Terrestrial solar spectral data sets," Solar Energy 30(6), 563-573 (1983).

[13] Arnulf, A., Bricard, J., Curé, E. and Véret, C., "Transmission by Haze and Fog in the Spectral Region 0.35 to 10 Microns*," J. Opt. Soc. Am. 47(6), 491-498 (1957).

[14] Tobin, R., Halimi, A., McCarthy, A., Laurenzis, M., Christnacher, F. and Buller, G. S., "Three-dimensional single-photon imaging through obscurants," Opt. Express 27(4), 4590-4611 (2019).

[15] Itzler, M. A., Entwistle, M., Owens, M., Patel, K., Jiang, X., Slomkowski, K., Rangwala, S., Zalud, P. F., Senko, T., Tower, J. and Ferraro, J., "Comparison of 32 x 128 and 32 × 32 Geiger-mode APD FPAs for single photon 3D LADAR imaging" Proc. SPIE 8033, 80330G (2011).

[16] Laurenzis, M., "Single photon range, intensity and photon flux imaging with kilohertz frame rate and high dynamic range," Opt. Express 27(26), 38391-38403 (2019).

[17] Legros, Q., Tachella, J., Tobin, R., McCarthy, A., Meignen, S., Buller, G. S., Altmann, Y., McLaughlin, S. and Davies, M. E., "Robust 3D reconstruction of dynamic scenes from single-photon lidar using Beta-divergences," arXiv:2004.09211 (2020).

[18] Pellegrini, S., Warburton, R. E., Tan, L. J. J., Ng, J. S., Krysa, A. B., Groom, K., David, J. P. R., Cova, S., Robertson, M. J. and Buller, G. S., "Design and performance of an InGaAs-InP single-photon avalanche diode detector," IEEE J. Quantum Electron. 42(4), 397-403 (2006).

[19] Campbell, J. C., Sun, W., Lu, Z., Itzler, M. A. and Jiang, X., "Common-Mode Cancellation in Sinusoidal Gating With Balanced InGaAs/InP Single Photon Avalanche Diodes,” IEEE J. Quantum Electron. 48(12), 1505-1511 (2012).

[20] Tosi, A., Calandri, N., Sanzaro, M. and Acerbi, F., "Low-Noise, Low-Jitter, High Detection Efficiency InGaAs/InP Single-Photon Avalanche Diode," IEEE J. Sel. Topics Quantum Electron. 20(6), 192-197 (2014).

[21]Zhang, J., Itzler, M. A., Zbinden, H. \& Pan, J.-W., "Advances in InGaAs/InP single-photon detector systems for quantum communication,” Light Sci. Appl. 4, e286 (2015).

[22] Comandar, L. C., Fröhlich, B., Dynes, J. F., Sharpe, A. W., Lucamarini, M., Yuan, Z. L., Penty, R. V. and Shields, A. J., "Gigahertz-gated InGaAs/InP single-photon detector with detection efficiency exceeding $55 \%$ at $1550 \mathrm{~nm}$," J. Appl. Phys. 117(8), 083109 (2015).

[23] Restelli, A., Bienfang, J. C. and Migdall, A. L., "Single-photon detection efficiency up to $50 \%$ at $1310 \mathrm{~nm}$ with an InGaAs/InP avalanche diode gated at 1.25 GHz,” Appl. Phys. Lett. 102(14), 141104 (2013). 
[24] Warburton, R. E., Intermite, G., Myronov, M., Allred, P., Leadley, D. R., Gallacher, K., Paul, D. J., Pilgrim, N. J., Lever, L. J. M., Ikonic, Z., Kelsall, R. W., Huante-Cerón, E., Knights, A. P. and Buller, G. S., "Ge-on-Si Single-Photon Avalanche Diode Detectors: Design, Modeling, Fabrication, and Characterization at Wavelengths 1310 and 1550 nm," IEEE Trans. Electron Devices 60(11), 3807-3813 (2013).

[25] Vines, P., Kuzmenko, K., Kirdoda, J., Dumas, D. C. S., Mirza, M. M., Millar, R. W., Paul, D. J. and Buller, G. S., "High performance planar germanium-on-silicon single-photon avalanche diode detectors," Nat. Commun. 10, 1086 (2019).

[26] Itzler, M. A., Jiang, X., Entwistle, M., Slomkowski, K., Tosi, A., Acerbi, F., Zappa, F. and Cova, S., "Advances in InGaAsP-based avalanche diode single photon detectors," J. Mod. Opt. 58(3-4), 174-200 (2011).

[27] Scarcella, C., Boso, G., Ruggeri, A. and Tosi, A., "InGaAs/InP Single-Photon Detector Gated at $1.3 \mathrm{GHz}$ With 1.5\% Afterpulsing," IEEE J. Sel. Topics Quantum Electron. 21(3), 17-22 (2015).

[28] Michel, J., Liu, J. and Kimerling, L. C., "High-performance Ge-on-Si photodetectors," Nat. Photonics 4, 527-534 (2010).

[29] Huang, M., Li, S., Cai, P., Hou, G., Su, T., Chen, W., Hong, C. and Pan, D., "Germanium on Silicon Avalanche Photodiode," IEEE J. Sel. Topics Quantum Electron. 24(2), 1-11 (2018).

[30] Virot, L., Crozat, P., Fédéli, J.-M., Hartmann, J.-M., Marris-Morini, D., Cassan, E., Boeuf, F. and Vivien, L., "Germanium avalanche receiver for low power interconnects," Nat. Commun. 5, 4957 (2014).

[31]Loudon, A. Y., Hiskett, P. A., Buller, G. S., Carline, R. T., Herbert, D. C., Leong, W. Y. and Rarity, J. G., "Enhancement of the infrared detection efficiency of silicon photon-counting avalanche photodiodes by use of silicon germanium absorbing layers," Opt. Lett. 27(4), 219-221 (2002).

[32] Lu, Z., Kang, Y., Hu, C., Zhou, Q., Liu, H.-D. and Campbell, J. C., "Geiger-Mode Operation of Ge-on-Si Avalanche Photodiodes,” IEEE J. Quantum Electron. 47(5), 731-735 (2011).

[33] Martinez, N. J. D., Gehl, M., Derose, C. T., Starbuck, A. L., Pomerene, A. T., Lentine, A. L., Trotter, D. C. and Davids, P. S., "Single photon detection in a waveguide-coupled Ge-on-Si lateral avalanche photodiode," Opt. Express 25(14), 16130-16139 (2017).

[34] Kuzmenko, K., Vines, P., Halimi, A., Collins, R. J., Maccarone, A., McCarthy, A., Greener, Z. M., Kirdoda, J., Dumas, D. C. S., Llin, L. F., Mirza, M. M., Millar, R. W., Paul, D. J., Buller, G. S., "3D LIDAR imaging using Ge-on-Si single photon avalanche diode detectors," Opt. Express 28(2), 1330-1344 (2020).

[35] Lacaita, A., Spinelli, A. and Longhi, S., "Avalanche transients in shallow p-n junctions biased above breakdown," Appl. Phys. Lett. 67(18), 2627-2629 (1995). 